\title{
Porcine reproductive and respiratory syndrome virus suppresses post-transcriptionally the protein expression of IFN- $\beta$ by upregulating cellular microRNAs in porcine alveolar macrophages in vitro
}

\author{
LILIN WANG ${ }^{1 *}$, LEI ZHOU ${ }^{1 *}$, DONGMEI HU ${ }^{2}$, XINNA GE $^{1}$, \\ XIN GUO ${ }^{1}$ and HANCHUN YANG ${ }^{1}$ \\ ${ }^{1}$ Key Laboratory of Animal Epidemiology of Ministry of Agriculture, College of Veterinary Medicine
and State Key Laboratory of Agrobiotechnology, China Agricultural University, Beijing 100193;
${ }^{2}$ Veterinary Diagnostic Laboratory, China Animal Disease Control Center, Beijing 102600, P.R. China
}

Received July 18, 2017; Accepted September 21, 2017

DOI: $10.3892 /$ etm.2017.5397

\begin{abstract}
Porcine reproductive and respiratory syndrome virus (PRRSV) has been recognized to inhibit the response of type I interferon (IFN) both in vivo and in vitro. However, the post-transcriptional mechanism by which PRRSV suppresses type I IFN induction in virus-infected host cells remains unclear. The present study first demonstrated that PRRSV inhibited post-transcriptionally the protein induction of IFN- $\beta$ in primary porcine alveolar macrophages (PAMs) during early infection, and the inhibition effect mediated by the Chinese highly pathogenic (HP)-PRRSV was stronger. Next, we analyzed the cellular microRNA (miRNA)-modulated protein expression of porcine IFN- $\beta$ by dual firefly/Renilla luciferase reporter assay, transfection of miRNA mimics and inhibitor assay and polyinosinic-polycytidylic acid (poly I:C) treatment of PAMs, showing that porcine miRNAs including let-7b, miR-26a, miR-34a and miR-145 are able to inhibit IFN- $\beta$ protein expression in primary PAMs by directly targeting sequences within the porcine IFN- $\beta$ 3'UTR locating at 160-181, 9-31, 27-47 and 12-32 bp, respectively. Finally, we confirmed that let-7b, miR-26a, miR-34a and miR-145, were upregulated in PRRSV-infected PAMs early in vitro, and the expression level of these miRNAs in HP-PRRSV JXwn06-infected PAMs
\end{abstract}

Correspondence to: Dr Hanchun Yang, Key Laboratory of Animal Epidemiology of Ministry of Agriculture, College of Veterinary Medicine and State Key Laboratory of Agrobiotechnology, China Agricultural University, 2 Yuanmingyan West Road, Haidian, Beijing 100193, P.R. China

E-mail: yanghanchun1@cau.edu.cn

${ }^{*}$ Contributed equally

Key words: porcine reproductive and respiratory syndrome virus, porcine alveolar macrophages, IFN- $\beta$, inhibition, microRNA were higher than those in low pathogenic PRRSV HB-1/3.9infected PAMs. The endogenous cellular miRNA-mediated inhibition of IFN- $\beta$ induction in PRRSV-infected PAMs early could be relieved by miRNA antagonists. Taken together, our findings suggest for the first time that PRRSV can suppress post-transcriptionally protein expression of IFN- $\beta$ by upregulating cellular miRNAs in PAMs in vitro, providing novel insight into mechanisms in relation to the PRRSV-mediated immunomodulation of porcine innate immunity.

\section{Introduction}

Porcine reproductive and respiratory syndrome (PRRS), which is characterized by reproductive disorders in pregnant sows and respiratory diseases in growing pigs, is a viral disease caused by PRRS virus (PRRSV) $(1,2)$. Since its first outbreak in 1987 in USA (3), PRRS has spread to the major pig-producing countries and regions (4), causing tremendous economic losses to the world pig industry. The emergence and prevalence of highly pathogenic (HP)-PRRSV with a 30 -aa discontinuous deletion in its nsp2-coding region devastated the Chinese swine industry in $2006(5,6)$. PRRSV, a single positive-stranded RNA virus, is classified into the genus Arterivirus of family Arteriviridae in the order Nidovirales (7-9). This virus can be divided into two genotypes, namely types 1 and 2 (10-12).

Studies have shown that PRRSV can utilize post-transcriptional control to inhibit the production of type I interferon (IFN) in its natural target cells $(13,14)$. PRRSV-infected target cells and host pig fail to induce the protein production of type I IFN. IFN- $\alpha$ protein was undetectable in lungs of PRRSV-infected pigs, and only a very small amount of IFN- $\alpha$ protein could be detected or was undetectable in the culture supernatant of PRRSV-infected peripheral blood mononuclear cells and monocyte dendritic cells in vitro (15-17). PRRSV cannot induce protein expression of IFN- $\alpha$ during viral infection, furthermore it blocks the production of IFN- $\alpha$ induced by porcine transmissible gastroenteritis virus (TGEV) or double-stranded RNA polyinosinic-polycytidylic acid (poly 
$\mathrm{I}: \mathrm{C})$ in vitro $(17,18)$. However, the mRNA transcription of $\alpha$ and IFN- $\alpha$ can be activated by PRRSV in host target cells in vitro and in vivo $(14,19,20)$. These findings clearly indicate that PRRSV suppresses the production of type I IFN in host by post-transcriptional and translational control. Although several previous studies exploring the molecular basis for PRRSV-mediated inhibition of type I IFN have demonstrated that PRRSV-encoded nsp 1, nsp 2, and nsp 11 and $\mathrm{N}$ protein play key roles in antagonizing the activation of IFN- $\beta$ promoter (21), these focused mainly on the stage of IFN- $\beta$ mRNA transcription, primarily in PRRSV permissive MARC-145 cells or non-permissive human cell culture systems. Therefore, these data have uncertain relevance to the host cells naturally infected with PRRSV. To date, the post-transcriptional mechanism concerning PRRSV-mediated innate immune response escape, especially blocking the production of type I IFN, during viral infection in host cells, remains unclear.

MicroRNAs (miRNAs) are small non-coding RNAs with approximately 22 nucleotides in length, which can bind to the miRNA seed sequence (2-8 nucleotides) complementarity motifs locating usually within the 3 '-untranslated region ( $3^{\prime} \mathrm{UTR}$ ) of the target mRNA to directly target gene silencing through mRNA cleavage, transcription degradation or translational inhibition (22). Although miRNAs can be induced or repressed directly by type I IFN, they play a crucial role in regulating the innate immune response including suppression or enhancement of type I IFN production (23). Moreover, during viral infection, not only can host cellular miRNAs target directly viral RNAs or regulate host antiviral genes to modify the cellular state for performing antiviral functions, but also cellular miRNAs can be manipulated by virus to facilitate viral replication $(24,25)$. Therefore, it is not surprising that PRRSV may interact with host cellular miRNAs to evade the type I IFN response. Actually, several previous studies have demonstrated that cellular miRNAs could be modulated during PRRSV infection (26-28), and some miRNAs could promote or inhibit PRRSV replication (29-32). Simultaneously, the mRNA transcription of type I IFN could be upregulated or downregulated by PRRSV-influenced cellular miRNAs (33-35), however, no literature on how PRRSV-modulating cellular miRNAs manipulate the protein expression of type I IFN have been documented.

Different strains of PRRSV have different sensitivity to IFN- $\alpha$, and possess different abilities to inhibit type I IFN induction (20), whilst cellular miRNA expression profile in porcine alveolar macrophages (PAMs) is also identified to be PRRSV strain-specific during viral infection (26). The inhibition of IFN- $\beta$ by PRRSV is of significant difference among different type of cells (36). PRRSV vaccine strain is insensitive to type I IFN in MARC-145 cells, however, in primary PAMs, PRRSV vaccine strain and virulent strain are sensitive to type I IFN. PRRSV has been shown to activate IFN- $\beta$ transcription in porcine monocyte-derived dendritic cells (14), but in MARC-145 cells, PRRSV inactivates and inhibits IFN- $\beta$ transcription activated by poly I:C (18). In addition to PRRSV strains and cell types, different pig breeds may also exhibit distinct characteristics during PRRSV infection. Compared with other pig breeds, PRRSV replication and proliferation can be suppressed or delayed in the PAMs of landrace pigs (37). Cellular miRNAs in lung tissues show significantly differential expression between tongcheng and landrace pigs during HP-PRRSV infection (27). Moreover, since the ultraviolet light- and heat-inactivated PRRSV cannot suppress type I IFN production induced by TGEV infection or poly I:C stimulation in PAMs, the inhibition of type I IFN by PRRSV in natural host cell may be pathogenicity-related (13). Additionally, the pathogenicity between HP-PRRSV and low pathogenic PRRSV strains are significantly different in vivo and in vitro, therefore, the question of whether HP-PRRSV and low pathogenic PRRSV show differences in the regulation and miRNA modulation of type I IFN during viral infection, is of considerable interest.

In the present study, we analyzed the inhibitory effect of PRRSV infection on the post-transcriptionally protein expression of porcine IFN- $\beta$, and further explored the relationship between cellular miRNAs and IFN- $\beta$ protein expression in primary PAMs.

\section{Materials and methods}

Cells and virus. Primary PAMs were prepared as previously described (38). PAM cell line 3D4/21 (CRL-2843) was obtained from the American Type Culture Collection (ATCC) (Manassas, VA, USA). The cells were grown in RPMI-1640 medium (Thermo Fisher Scientific, Inc., Waltham, MA, USA) supplemented with $10 \%$ fetal bovine serum (Hyclone Laboratories Inc., Logan, UT, USA) at $37^{\circ} \mathrm{C}$, with $5 \% \mathrm{CO}_{2}$. The HP-PRRSV JXwn06 (GenBank accession no. EF641008) and low pathogenic PRRSV HB-1/3.9 (GenBank accession no. EU360130) were used in this study (39).

miRNA target predictions. To analyze the interaction between cellular miRNAs and porcine IFN- $\beta$ 3'UTR, we selected a serial of miRNAs, especially porcine let-7b, miR-26a, miR-34a and miR-145 obtained from miRBase (http://www.mirbase. org), and predicted miRNA-targeted binding sequences in porcine IFN- $\beta$ 3'UTR, whose sequences were from GenBank (http://www.ncbi.nlm.nih.gov/GenBank), by miRanda (40), RNA hybrid (41) and PITA (42).

miRNA mimics and miRNA inhibitors. Based on the sequences of porcine cellular miRNAs, including let-7b, miR-26a, miR-34a and miR-145, miRNA mimics and miRNA inhibitors and negative control were designed (Table I), and were synthesized with 2'-O-methyl modification by Shanghai GenePharma Co., Ltd. (Shanghai, China).

Plasmid construction. Three plasmids including pmirGLO-IFN- $\beta$ 3'UTR, pmirGLO- miRNA recognition elements (MRE)-wild type (wt) and pmirGLO-MRE-mutant type (mut) were constructed based on the pmirGLO dual-luciferase miRNA target expression vector (Promega Corp., Madison, WI, USA) following the manufacturer's instructions.

To construct pmirGLO-IFN- $\beta$ 3'UTR, the PCR amplification primers were designed by Primer Premier v5.0 according to the sequence of porcine IFN- $\beta$ 3'UTR (GenBank accession no. M86762). PmeI and XbaI restriction enzyme sites (underlined letters) were introduced in the upstream forward primer (5'-ATAGTTTAAACACATCTCCCCCCTGTGGCT-3') and 
Table I. The sequences of the synthesized porcine miRNA mimics and miRNA inhibitors.

\begin{tabular}{lll}
\hline miRNA & \multicolumn{1}{c}{ miRNA mimic sequence $\left(5^{\prime} \rightarrow 3^{\prime}\right)$} & miRNA inhibitor sequence $\left(5^{\prime} \rightarrow 3^{\prime}\right)$ \\
\hline let-7b & UGAGGUAGUAGGUUGUGUGGUU & AACCACACAACCUACUACCUCA \\
miR-26a & UUCAAGUAAUCCAGGAUAGGCU & AGCCUAUCCUGGAUUACUUGAA \\
miR-34a & UGGCAGUGUCUUAGCUGGUUGU & ACAACCAGCUAAGACACUGCCA \\
miR-145 & GUCCAGUUUUCCCAGGAAUCCCUU & AAGGGAUUCCUGGGAAAACUGGAC \\
NC & UUGUACUACACAAAAGUACUG & CAGUACUUUUGUGUAGUACAA
\end{tabular}

NC, negative control.

Table II. Primers for wild-type and mutant-type MRE in porcine IFN- $\beta$ 3'UTR.

\begin{tabular}{ll}
\hline Primer $^{\text {a }}$ & \multicolumn{1}{c}{ Primer sequence $\left(5^{\prime} \rightarrow 3^{\prime}\right)^{\mathrm{b}}$} \\
\hline let-7b MRE-F & AAACTAGCGGCCGCTAGTATGTATTTAATTTTTACCTTGT \\
let-7b MRE-R & CTAGACAAGGTAAAAAATTAAATACATACTAGCGGCCGCTAGTTT \\
let-7b Mut-F & AAACTAGCGGCCGCTAGTATGTATTTAATTTGATGGAGGT \\
let-7b Mut-R & CTAGACCTCCATCAAAATTAAATACATACTAGCGGCCGCTAGTTT \\
miR-26a MRE-F & AAACTAGCGGCCGCTAGTCCCCTGTGGCTCTGGGAATTGACT \\
miR-26a MRE-R & CTAGAGTCAATTCCCAGAGCCACAGGGGACTAGCGGCCGCTAGTTT \\
miR-26a Mut-F & AAACTAGCGGCCGCTAGTCCCCTGTGGCTCTGGGTGAACTCT \\
miR-26a Mut-R & CTAGAGAGTTCACCCAGAGCCACAGGGGACTAGCGGCCGCTAGTTT \\
miR-34a MRE-F & AAACTAGCGGCCGCTAGTTTGACCATGTTGGCAATGATGT \\
miR-34a MRE-R & CTAGACATCATTGCCAACATGGTCAAACTAGCGGCCGCTAGTTT \\
miR-34a Mut-F & AAACTAGCGGCCGCTAGTTTGACCATGTTGGGTGACGGGT \\
miR-34a Mut-R & CTAGACCCGTCACCCAACATGGTCAAACTAGCGGCCGCTAGTTT \\
miR-145 MRE-F & AAACTAGCGGCCGCTAGTCTGTGGCTCTGGGAATTGACCT \\
miR-145 MRE-R & CTAGAGGTCAATTCCCAGAGCCACAGACTAGCGGCCGCTAGTTT \\
miR-145 Mut-F & AAACTAGCGGCCGCTAGTCTGTGGCTCTGGGTTGACCTCT \\
miR-145 Mut-R & CTAGAGAGGTCAACCCAGAGCCACAGACTAGCGGCCGCTAGTTT
\end{tabular}

${ }^{\mathrm{a}} \mathrm{F}$ and R, primer pairs were annealed and inserted into pmirGLO dual-luciferase miRNA target expression vector digested with $P m e \mathrm{I}$ and $\mathrm{XbaI}$; ${ }^{\mathrm{b}} N o t \mathrm{I}$ restriction endonuclease site is underlined; MRE, miRNA recognition elements; IFN- $\beta$, interferon- $\beta$; $3^{\prime}$ UTR, $3^{\prime}$ untranslated region.

downstream reverse primer (5'-TGCTCTAGAGGATCCT TAACCCACTGATCCAG-3'), respectively. Taken PAMs genomic DNA as template, PCR was performed to generate porcine IFN- $\beta$ 3'UTR by high-fidelity PrimeSTAR ${ }^{\circledR}$ HS DNA Polymerase (Takara Biotechnology Co., Ltd., Dalian, China) according to manufacturer's instructions. After digested with PmeI and $\mathrm{XbaI}$, both porcine IFN- $\beta$ 3'UTR gene fragments and pmirGLO dual firefly/Renilla luciferase miRNA target expression vector were ligated together by T4 DNA ligase (Promega Corp.) following the manufacturer's protocol.

To generate pmirGLO-MRE-wt and pmirGLO-MRE-mut, according to the predicted MRE in porcine IFN- $\beta$ 3'UTR for let-7b, miR-26a, miR-34a and miR-145, the DNA fragments of wild-type MRE and mutant-type MRE (base mutations in the seed region) with NotI restriction endonuclease sites were prepared by annealing the primer pairs listed in Table II under the following conductions: $94^{\circ} \mathrm{C} 4 \mathrm{~min}, 70^{\circ} \mathrm{C} 10 \mathrm{sec}$, $65^{\circ} \mathrm{C} 10 \mathrm{sec}, 60^{\circ} \mathrm{C} 10 \mathrm{sec}, 58^{\circ} \mathrm{C} 10 \mathrm{sec}, 55^{\circ} \mathrm{C} 10 \mathrm{sec}, 55^{\circ} \mathrm{C}$ water bath to cool for $90 \mathrm{~min}$, and then the resulting product DNA fragments were cloned into the pmirGLO dual firefly/Renilla luciferase miRNA target expression vector previously digested with PmeI and $\mathrm{XbaI}$, by T4 DNA ligase according to manufacturer's instructions. All plasmids were verified by digesting with NotI and sequencing.

Transient transfection and PRRSV infections. The 3D4/21 cells were seeded in 24-well cell culture plates and incubated overnight until the cell density reached $70-80 \%$, then transfected by FuGENE ${ }^{\circledR}$ HD Transfection Reagent (Promega Corp.) according to manufacturer's instructions using the modified method in our recent study (43). Briefly, $500 \mu 1$ serum and antibiotic-free opti-MEM were added in sterile $1.5 \mathrm{ml}$ centrifuge tube, then FuGENE ${ }^{\circledR}$ HD transfection reagent was added in accordance with the proportion required, and mixed gently and placed at room temperature for $15 \mathrm{~min} ; 0.5 \mu \mathrm{g}$ plasmid DNA and miRNA mimics at a final concentration of 10 or 40 or $60 \mathrm{nM}$, were added and mixed gently and placed at room temperature for $30 \mathrm{~min}$. The mixture was given to 
the culture plates, and then mixed gently, treated at $37^{\circ} \mathrm{C}$ in a humidified incubator with $5 \% \mathrm{CO}_{2}$ for $24 \mathrm{~h}$.

Primary PAMs with a density of about $0.5-1 \times 10^{5}$ cells per $\mathrm{ml}$ were seeded in 24-well culture plates, cultured at $37^{\circ} \mathrm{C}$ in a humidified incubator with $5 \% \mathrm{CO}_{2}$ overnight. After changing to fresh medium, the cells were transfected transiently with miRNA mimics and inhibitors by HiPerFect ${ }^{\circledR}$ transfection reagent (Qiagen China Co., Ltd., Shanghai, China) according to manufacturer's instructions as previously described (44). Briefly, miRNA mimics at a final concentration of 10 or $40 \mathrm{nM}$, or miRNA inhibitors at 20 or 40 or $100 \mathrm{nM}$ were added in $100 \mu \mathrm{l}$ serum and antibiotic-free opti-MEM in sterile $1.5 \mathrm{ml}$ centrifuge tube, and then the HiPerFect ${ }^{\circledR}$ transfection reagent in corresponding volume ratio following the instructions was added, and treated at room temperature for 5-10 min after being mixed. The mixture was given to the culture plates after shaking gently, cultured at $37^{\circ} \mathrm{C}$ in a humidified incubator with $5 \% \mathrm{CO}_{2}$.

For viral infections, prepared primary PAMs were seeded in 6-well culture plates, and divided into three groups including JXwn06-infected group, HB-1/3.9-infected group and mock-infected group as control. In PRRSV-infected groups, PAMs were inoculated with JXwn06 or HB-1/3.9 at MOI of 0.01 or 1 , and then cultured at $37^{\circ} \mathrm{C}$ in a humidified incubator with $5 \% \mathrm{CO}_{2}$. Cell samples and supernatant samples were collected at $0 \mathrm{~h}$ post-infection (hpi), 12, 24, and $36 \mathrm{hpi}$ to detect the RNA expressions by real-time PCR and IFN- $\beta$ protein expression by ELISA, respectively.

Dual luciferase reporter assay. After 3D4/21 cells were co-transfected transiently with miRNA mimics and plasmid DNA (pmirGLO-IFN- $\beta$ 3'UTR orpmirGLO-MRE-wt or pmirGLO-MRE-mut) for $24 \mathrm{~h}$, dual luciferase activity reporter assay was performed using dual-luciferase ${ }^{\circledR}$ reporter assay system (Promega Corp.) according to manufacturer's instructions. Briefly, the cell culture medium in culture plates was removed, and washed twice with PBS. To lyse cells fully, passive lysis buffer was added according to its protocol, and treated at room temperature for $20 \mathrm{~min}$. The lysate was transferred into a 96-well plate, and then the plate was placed at luciferase detector GloMAX ${ }^{\mathrm{TM}} 96$ microplate luminometer (Promega Corp.) to detect firefly luciferase activity and Renilla luciferase activity. The data are shown as the ratio of firefly luciferase activity value to Renilla luciferase activity value for quantitative analysis of luciferase activity. Each experiment was repeated three times.

$R N A$ isolation and $q R T-P C R$. To extract total RNAs (including mRNA and miRNA) from primary PAMs, the prepared cell samples were lysed by TRIzol reagent (TransGen Biotech, Inc., Beijing, China) following the manufacturer's instructions. Total RNAs extracted from PAMs were digested by RQ1 RNase-Free DNase (Promega Corp.) to remove possible residual DNA according to the manufacturer's protocol. The RNAs concentration was determined, and total RNAs were subjected immediately to reverse transcription or stored at $-80^{\circ} \mathrm{C}$ for use.

For detection of IFN- $\beta$ mRNA, $1 \mu \mathrm{g}$ purified total RNAs and $0.5 \mu \mathrm{g}$ random primer hexa-deoxyribonucleotide mixture (Takara Biotechnology Co., Ltd.) was mixed together, and then placed in water bath at $70^{\circ} \mathrm{C}$ for 5 min, immediately cooled on ice for $5 \mathrm{~min}$, the product was added to the following reaction components for reverse transcription: $4 \mu 15 \mathrm{x}$ M-MLV reaction buffer, $1 \mu \mathrm{l} 10 \mathrm{mM}$ dNTPs, $0.5 \mu \mathrm{l} 40 \mathrm{U} / \mu \mathrm{l}$ RNase inhibitor, $1 \mu \mathrm{l}$ of $10 \mathrm{U} / \mu \mathrm{l}$ M-MLV reverse transcriptase (Promega Corp.), RNase-free $\mathrm{ddH}_{2} \mathrm{O}$ was added to $20 \mu \mathrm{l}$. The mixture was incubated in water bath at $37^{\circ} \mathrm{C}$ for $1 \mathrm{~h}$, and then cDNA template was obtained. The cDNA template was taken and diluted in the proportion of 1:20, quantitative real-time PCR (qRT-PCR) was performed using 7500 Real Time PCR system (Applied Biosystems; Thermo Fisher Scientific, Inc.) and SYBR ${ }^{\circledR}$ Green real-time PCR master mix (Toyobo Life Science, Osaka, Japan) according to manufacturer's instructions with the primers for porcine IFN- $\beta$ (45) and RPL4 (46) (Table III). The reaction was in a $20 \mu \mathrm{l}$ volume containing $10 \mu \mathrm{l} 2 \mathrm{x} \mathrm{SYBR}^{\circledR}$ Green Real Time PCR Master Mix, $0.8 \mu 110 \mathrm{pmol} / \mu 1$ primer pair, $1 \mu \mathrm{l}$ cDNA template, and 8.2 $\mu \mathrm{l}$ RNase-free $\mathrm{ddH}_{2} \mathrm{O}$. The PCR conditions comprised an initial pre-denaturation at $95^{\circ} \mathrm{C}$ for $1 \mathrm{~min}$, and 40 cycles of $95^{\circ} \mathrm{C}$ for $15 \mathrm{sec}, 65^{\circ} \mathrm{C}$ for $15 \mathrm{sec}, 72^{\circ} \mathrm{C}$ for $45 \mathrm{sec}$. To assess the specificity of PCR products, melting curve analysis and size verification were performed. Relative expression level of IFN- $\beta$ gene was analyzed using the $\Delta \Delta \mathrm{Ct}$ method and the housekeeping gene RPL4 mRNA was used as an internal control.

For detection of miRNAs, $1 \mu \mathrm{g}$ of the purified total cellular RNAs was used for polyadenylation using E. coli Poly (A) polymerase (New England BioLabs Inc., Ipswich, MA, USA) according to manufacturer's instructions. To obtain cDNA template of miRNAs, the polyadenylated total cellular RNAs were reverse-transcribed with a universal adapter primer (miRNA RT Primer) or U6 downstream primer (U6-R) (Table III) using M-MLV reverse transcriptase in accordance with manufacturer's instructions. Based on miRNAs sequences in miRBase, the specific primers for porcine let-7b, miR-26a, miR-34a, miR-145 and internal reference U6, were designed and synthesized (Table III). Quantitative real-time PCR were performed with the primers for miRNAs using 7500 Real Time PCR system (Applied Biosystems; Thermo Fisher Scientific, Inc.) and SYBR ${ }^{\circledR}$ Green Real Time PCR Master Mix (Toyobo Life Science) in accordance with manufacturer's instructions, the specificity of PCR products were assessed as described above. The relative expression levels of miRNAs were normalized internally utilizing U6 as a reference using the $\Delta \Delta \mathrm{Ct}$ method as previously described (44).

ELISA for porcine IFN- $\beta$. To determine the protein induction of IFN- $\beta$ in culture supernatants of primary PAMs, porcine IFN- $\beta$ detection was performed using a commercial ELISA kit (DZE40056; DongGe Biotechnology, Beijing, China) according to manufacturer's instructions. After the cell concentration was adjusted, the prepared primary PAMs were seeded in six-well cell culture plates incubated in $10 \%$ RPMI-1640 medium at $37^{\circ} \mathrm{C}$ in a humidified cell incubator with $5 \% \mathrm{CO}_{2}$. Non-adherent cells were removed at about $2.5 \mathrm{~h}$ after the incubation, and then complete growth medium was added to continue incubating overnight. Primary PAMs were infected with PRRSV JXwn06 or HB-1/3.9, or transfected transiently using miRNA mimics or inhibitors, or cultured in fresh 10\% RPMI-1640 growth medium containing a final concentration of $50 \mu \mathrm{g} / \mathrm{ml}$ poly I:C (Sigma-Aldrich, Inc., 
Table III. Primers for reverse transcription and real-time PCR of porcine IFN- $\beta$ and miRNAs.

\begin{tabular}{ll}
\hline Primer $^{\mathrm{a}}$ & \multicolumn{1}{c}{ Primer sequence $\left(5^{\prime} \rightarrow 3^{\prime}\right)^{\mathrm{b}}$} \\
\hline IFN- $\beta-\mathrm{F}$ & TGCAACCACCACAATTCC \\
IFN- $\beta-\mathrm{R}$ & CTGAGAATGCCGAAGATCTG \\
RPL4-F & CAAGAGTAACTACAACCTTC \\
RPL4-R & GAACTCTACGATGAATCTTC \\
miRNA RT primer & GCGAGCACAGAATTAATACGACTCACTATAGGT \\
let-7b-F & CGGTGAGGTAGTAGGTTGTGTGGTT \\
miR-26a-F & CGGTTCAAGTAATCCAGGATAGGCT \\
miR-34a-F & GGTGGCAGTGTCTTAGCTGGTTGT \\
miR-145-F & GTCCAGTTTTCCCAGGAATCCCT \\
Uni-miRNA-R & GCGAGCACAGAATTAATACGACTCAC \\
U6-F & CTCGCTTCGGCAGCACA \\
U6-R & AACGCTTCACGAATTTGCGT
\end{tabular}

${ }^{\mathrm{a}} \mathrm{F}$, forward PCR primer; R, reverse PCR primer except U6-R acted as reverse transcription primer and reverse PCR primer; ${ }^{b} \mathrm{~V}, \mathrm{G} / \mathrm{A} / \mathrm{C}$; N, $\mathrm{A} / \mathrm{G} / \mathrm{C} / \mathrm{T}$.

St. Louis, MO, USA), meanwhile mock-infected or negative control-transfected or mock-treated cells acted as control group, respectively. The cell culture supernatant samples were harvested at different time points and used for detection of porcine IFN- $\beta$ protein by ELISA. The corresponding IFN- $\beta$ concentration in samples was obtained by their OD values according to the standard curve following manufacturer's instructions. The data are shown as relative expression levels of IFN- $\beta$ protein normalized to control group.

Statistical analysis. Experimental data are presented as means \pm standard error of the mean. Significant differences of the variability among different groups were analyzed by two-way ANOVA test or t-test using GraphPad Prism (version 5.0; GraphPad Software, Inc., La Jolla, CA, USA) software. A $\mathrm{P}<0.05$ was considered to indicate a statistically significant difference.

\section{Results}

PRRSV inhibits post-transcriptionally the protein production of IFN- $\beta$ in primary PAMs. To analyze the differences in mRNA transcription and protein expression of IFN- $\beta$ in PAMs following PRRSV infection, primary PAMs were infected with JXwn06 or HB-1/3.9 at MOI of 0.01 or 1 , while mock-infected cells served as negative control. Cell samples and supernatant samples were collected at 0,12, 24 and 36 hpi. Cell samples were used to analyze the mRNA transcription level of IFN- $\beta$ by real-time PCR. Supernatant samples were subjected to detection of the protein concentration of IFN- $\beta$ by ELISA kit. The results showed that compared with mock group, IFN- $\beta$ mRNA transcription levels increased from 12 to 36 hpi both in JXwn06- and HB-1/3.9-infected PAMs (Fig. 1A), indicating that mRNA transcription of porcine IFN- $\beta$ was upregulated in primary PAMs early infected with PRRSV.

The transcription levels of porcine IFN- $\beta$ at MOI of 1 were higher than that at MOI of 0.01 in both JXwn06- and
HB-1/3.9-infected PAMs, showing that the regulation effect of PRRSV on porcine IFN- $\beta$ mRNA transcription is dose-dependent in primary PAMs infected with PRRSV. IFN- $\beta$ mRNA transcription levels in JXwn06-infected PAMs were higher compared with HB-1/3.9-infected PAMs. At MOI of 0.01, the transcription levels of porcine IFN- $\beta$ mRNA were significantly different at 36 hpi between JXwn06- and HB-1/3.9-infected PAMs $(\mathrm{P}<0.001)$. At MOI of 1 , the transcription levels of porcine IFN- $\beta$ mRNA were significantly different between the two strains of PRRSV at $12(\mathrm{P}<0.05)$ and $24 \mathrm{hpi}(\mathrm{P}<0.001)$, respectively (Fig. 1A). These data indicated that porcine IFN- $\beta$ mRNA transcription exhibited stronger upregulation effect in HP-PRRSV JXwn06-infected PAMs in vitro than that in low pathogenic PRRSV HB-1/3.9-infected PMAs at the same initial viral infectious dose during early infection.

On the contrary, normalized to negative control group at each time point, the protein relative expression levels of IFN- $\beta$ in PRRSV-infected PAMs were downregulated (Fig. 1B), suggesting that IFN- $\beta$ protein expression was inhibited by PRRSV in primary PAMs during early infection. The concentration of IFN- $\beta$ protein in the culture supernatants of negative control PAM cells increased higher than that in the culture supernatants of PRRSV-infected PAM cells at each time point, although the absolute concentration of IFN- $\beta$ protein in the culture supernatants of PRRSV-infected PAM cells augmented very little as time went on during early viral infection, the relative concentration of IFN- $\beta$ protein normalized to negative control decreased. To better analyze the relationship between PRRSV infection and IFN- $\beta$ protein expression and better observe the variation in the IFN- $\beta$ protein expression levels of PRRSV-infected PAMs, the IFN- $\beta$ protein data were shown as relative expression levels normalized to negative control group at each time point (Fig. 1B). The relative protein expression levels of porcine IFN- $\beta$ at MOI of 1 were lower than that at MOI of 0.01 in both JXwn06- and HB-1/3.9-infected PAMs, indicating that the PRRSV-mediated inhibition of porcine IFN- $\beta$ protein relative 

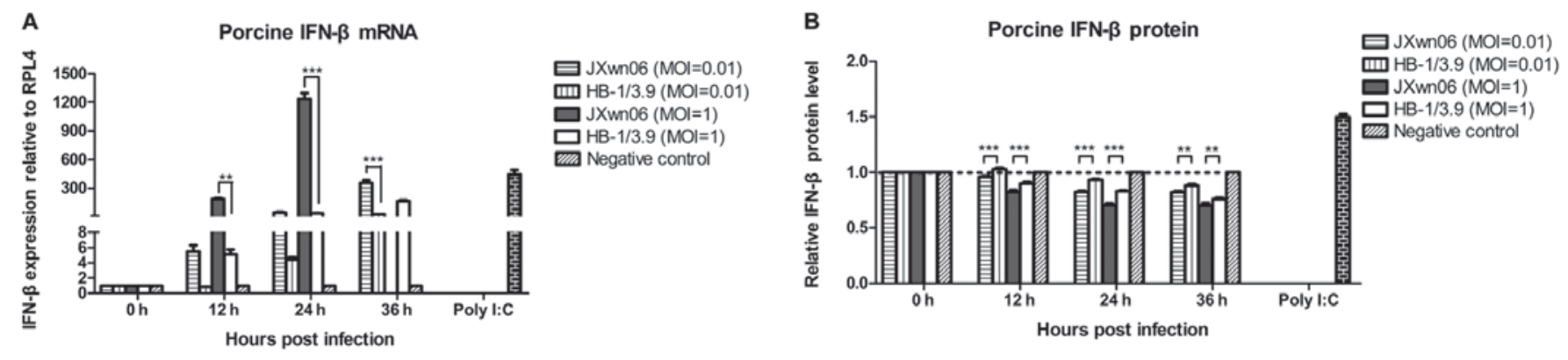

Figure 1. Inhibition of the IFN- $\beta$ protein expression in PRRSV-infected PAMs by post-transcriptional control. (A) The mRNA levels of porcine IFN- $\beta$ in PRRSV-infected PAMs and (B) its protein expression levels in the culture supernatants of PRRSV-infected PAMs were detected by real-time PCR and ELISA, respectively. Primary PAMs were infected with JXwn06 or HB-1/3.9 at MOI of 0.01 or 1, and then the cell and supernatant samples harvested at $0,12,24$ and $36 \mathrm{hpi}$, were measured by real-time PCR and ELISA, respectively, while mock-infected primary PAMs served as negative control and primary PAMs stimulated by $50 \mu \mathrm{g} / \mathrm{ml}$ poly I:C for $24 \mathrm{~h}$ were used as positive control. Relative expression level of IFN- $\beta$ gene was analyzed using the $\Delta \Delta \mathrm{Ct}$ method and the housekeeping gene RPL4 mRNA was used as an internal control. The IFN- $\beta$ protein data are shown as relative expression levels normalized to negative control group at each time point, respectively. The data represent means \pm standard error of the mean of three independent experiments $\left({ }^{* *} \mathrm{P}<0.01 ;{ }^{* * *} \mathrm{P}<0.001\right)$. Since the primary PAMs infected with JXwn06 at MOI of 1 were disrupted at $36 \mathrm{hpi}$, the data in relation to IFN- $\beta$ mRNA transcription could not be analyzed. IFN- $\beta$, interferon- $\beta$.

expression in primary PAMs is in dose-dependent manner during early infection. The downregulation of IFN- $\beta$ protein relative expression in JXwn06-infected PAMs was more significant than that in HB-1/3.9-infected PAMs (Fig. 1B) $(\mathrm{P}<0.05)$, suggesting that the inhibition effect of porcine IFN- $\beta$ protein expression by HP-PRRSV was stronger. Although it seemed to be small variation in porcine IFN- $\beta$ protein production in PAMs among different treated groups, it still made biological sense, because the succedent signaling cascades would amplify the IFN- $\beta$ effect thousands of times (47).

As a whole, the above results demonstrated that mRNA transcription levels of IFN- $\beta$ in primary PAMs early infected with PRRSV were upregulated, whereas its protein relative expression levels were downregulated, suggesting that PRRSV infection suppresses the protein expression of IFN- $\beta$ in PAMs by post-transcriptional control, and HP-PRRSV has stronger effect on the downregulation of IFN- $\beta$ protein relative expression than low pathogenic PRRSV at the same initial viral infectious dose during early infection in PAMs in vitro.

Porcine let-7b, miR-26a, miR-34a and miR-145 could inhibit protein expression of IFN- $\beta$ in primary PAMs by directly targeting sequences within porcine IFN- $\beta 3^{\prime} U T R$.

To understand the post-transcriptional molecular basis of the porcine IFN- $\beta$ protein expression in PAMs, the roles of porcine miRNAs were investigated. Since some miRNAs had been demonstrated to regulate IFN- $\beta$ protein expression in primary primate macrophages (47), to predict the interaction between cellular miRNAs and porcine IFN- $\beta 3$ 'UTR in primary PAMs, the binding sequences targeted by porcine miRNAs including let-7b, miR-26a, miR-34a and miR-145 within IFN- $\beta$ 3'UTR were analyzed using miRanda, RNA hybrid and PITA. The results showed that the cellular miRNAs let-7b, miR-26a, miR-34a and miR-145, could target porcine IFN- $\beta$ 3'UTR, and the binding sequences were located at 160-181, 9-31, 27-47 and $12-32 \mathrm{bp}$ in porcine IFN- $\beta$ 3'UTR, respectively. The specific interacting keys between these miRNAs and IFN- $\beta$ 3'UTR are shown in Fig. 2A.
By using pmirGLO-IFN- $\beta$ 3'UTR, pmirGLO-MRE-wt and pmirGLO-MRE-mut, the interactions between miRNAs and porcine IFN- $\beta$ 3'UTR were verified. The 3D4/21 cells were co-transfected transiently with pmirGLO-IFN- $\beta$ 3'UTR and a final concentration of 10 or $40 \mathrm{nM}$ miRNA mimics, and dual luciferase activity reporter assay was performed by dual-luciferase ${ }^{\circledR}$ reporter assay system. As shown in Fig. 2B, miRNA mimics could inhibit the expression of firefly luciferase in pmirGLO-IFN- $\beta$ 3'UTR. Compared with the control miRNA (CmiR), relative luciferase activity was suppressed by miRNA mimics, including let-7b, miR-26a, miR-34a and miR-145 at $10 \mathrm{nM}$, while at $40 \mathrm{nM}$ the inhibition of four miRNAs was stronger. The 3D4/21 cells were co-transfected with pmirGLO-MRE-wt or pmirGLO-MRE-mut and miRNA mimics at $60 \mathrm{nM}$, dual luciferase activity reporter assay was then performed. The results showed that miRNA mimics inhibited the expression of firefly luciferase of pmirGLO-MRE-wt, whereas the inhibition of firefly luciferase expression by miRNA was weakened for pmirGLO-MRE-mut. The relative luciferase activity ratio between pmirGLO-MREs-mut and pmirGLO-MREs-wt was greater than 1 (Fig. 2C). These results suggested that let-7b, miR-26a, miR-34a and miR-145 were capable of interacting with the predicted miRNA binding sequence in porcine IFN- $\beta$ 3'UTR, resulting in the protein expression inhibition of porcine IFN- $\beta$.

There is a mutual regulation between miRNAs and IFN- $\beta$ protein expression in PAMs. The miRNA-regulating protein expression of IFN- $\beta$ in primary PAMs was analyzed. By using miRNA HiPerFect ${ }^{\circledR}$ transfection reagent, the primary PAMs were transfected transiently with a final concentration of 10 or $40 \mathrm{nM}$ miRNAs mimics, including let-7b, miR-26a, miR-34a, miR-145 and CmiR, or a final concentration of 20 or $100 \mathrm{nM}$ miRNA inhibitors including four miRNA inhibitors and negative control (CmiRi). At about $3.5 \mathrm{~h}$ post-transfection, a final concentration of $50 \mu \mathrm{g} / \mathrm{ml}$ poly I:C was used to stimulate the PAMs for $24 \mathrm{~h}$, cell culture supernatant samples were then subjected to detect porcine IFN- $\beta$ protein expression by ELISA kit. As shown in Fig. 3A and B, compared with the 
A

Porcine IFN- $\beta$ 3'UTR (160-181 bp): $5^{\prime}$ AUGUAUUUAAUUUUUUACCUUG $3^{\prime}$ | : : : : :||::|: :||||||: let-7b: 3 ' UUGGUGUGUUGGAUGAUGGAGU 5 '

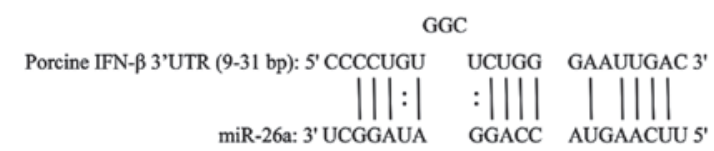

UA

$\mathrm{U}$

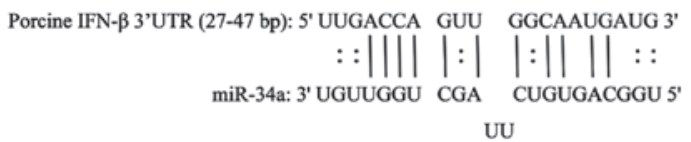

Porcine IFN- $\beta$ 3'UTR (12-32 bp): $5^{\prime}$ CUGUGGCU CUGGG AAUUGACC $3^{\prime}$

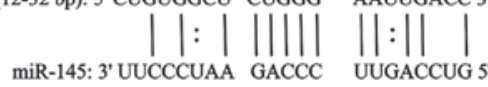

uU

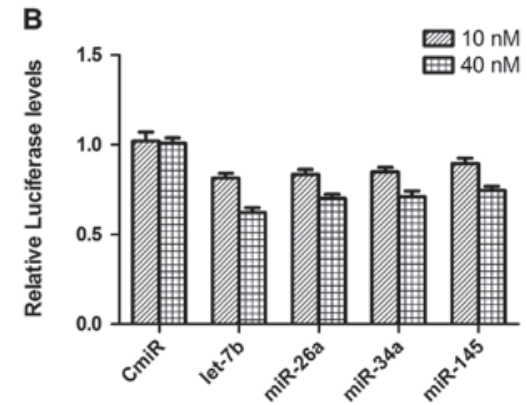

C

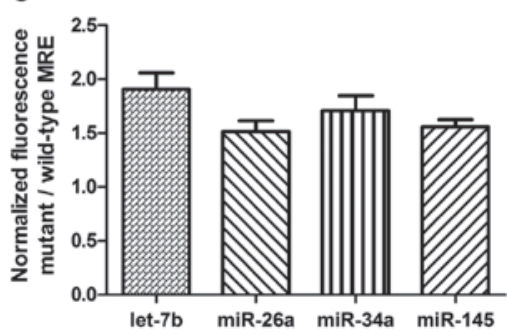

Figure 2. miRNAs target sequences located in the porcine IFN- $\beta$ 3'UTR. miRNA recognition elements (MRE) in the porcine IFN- $\beta$ 3'UTR for porcine let-7b, miR-26a, miR-34a and miR-145, were predicted using miRanda, RNA hybrid and PITA. (A) Porcine IFN- $\beta$ 3'UTR gene was cloned into the downstream of firefly luciferase in pmirGLO dual firefly/Renilla luciferase miRNA target expression vector, and the expression of pmirGLO-IFN- $\beta$ 3'UTR luciferase was inhibited in 3D4/21 cells transfected with miRNA mimics at a final concentration of 10 or $40 \mathrm{nM}$, (B) 3D4/21 cells were co-transfected with pmirGLO-MRE-wtor pmirGLO-MRE-mut and miRNA mimics at $60 \mathrm{nM}$, the fluorescence results are shown as the ratio of $\mathrm{mt}$ to wt MREs (C) the data represent means \pm standard error of the mean of three independent experiments. IFN- $\beta$, interferon- $\beta ; 3^{\prime}$ UTR, $3^{\prime}$ untranslated region; MRE, miRNA recognition elements; mt, mutant type; wt, wild-type.

negative control group, miRNA mimics including let-7b, miR-26a, miR-34a and miR-145, were capable of inhibiting the protein production of porcine IFN- $\beta$ in primary PAMs at $10 \mathrm{nM}$, while at $40 \mathrm{nM}$ the inhibition effect of four miRNAs were significantly stronger. In contrast, compared with the negative control group, except for the miR-145, three of the four miRNA inhibitors, were able to weaken the suppression effect of porcine cellular endogenous miRNAs in primary PAMs on IFN- $\beta$ protein expression at $20 \mathrm{nM}$, while at $100 \mathrm{nM}$ the relieving effect of four miRNA inhibitors were significantly stronger.

The native IFN- $\beta$ protein-regulating expression of cellular endogenous miRNAs in primary PAMs was further analyzed. Cell culture supernatant samples and cell samples were collected at $1,3,8$ and $24 \mathrm{~h}$ after poly I:C treatment, respectively. The protein expression levels of IFN- $\beta$ in the supernatants were measured by ELISA kit, miRNAs including let-7b, miR-26a, miR-34a and miR-145 in the cell samples were detected by real-time PCR using $\Delta \Delta \mathrm{Ct}$ method. As shown in Fig. $3 \mathrm{C}$, the expression levels of porcine IFN- $\beta$ protein were increased subsequently at $3 \mathrm{~h}$ after primary PAMs were stimulated by poly I:C. As shown in Fig. 3D, compared with untreated cells, the levels of native endogenous miRNAs in primary PAMs were increased with varying degrees (normalized to U6 expression and mock-treated controls). Taken together, native IFN- $\beta$ protein expression and cellular endogenous miRNAs could be induced by poly I:C together, and cellular endogenous miRNAs were increased only at or after the first augmentation of native IFN- $\beta$ protein induction in primary PAMs, indicating that the increasing porcine native IFN- $\beta$ protein could lead to the upregulation of the endogenous miRNA levels. Furthermore, the miR-26a and miR-34a had a significant increase, and let-7b was initially increased, and then decreased; the miR-145 showed an increase at a low level; the let-7b and miR-26a were upregulated significantly in the early stage of IFN- $\beta$ protein expression at a low level, demonstrating that it was exquisite for native IFN- $\beta$ protein to modulate the expression of cellular endogenous miRNAs in primary PAMs.

In short, the four cellular miRNAs could modulate the protein induction of IFN- $\beta$ in PAMs, meanwhile, the native IFN- $\beta$ induced in PAMs could also regulate the expression of four endogenous miRNAs, suggesting that a negative feedback loop might exist in the regulation of porcine IFN- $\beta$ protein on the expression of cellular miRNAs including let-7b, miR-26a, miR-34a and miR-145.

PRRSV infection suppresses IFN- $\beta$ protein expression in PAMs by upregulating cellular miRNAs. In order to investigate the modulation effect of the four cellular miRNAs during PRRSV infection, the levels of miRNAs in PRRSV JXwn06-infected and HB-1/3.9-infected primary PAMs were detected by real-time PCR. The results showed that the cellular miRNAs including let-7b, miR-26a, miR-34a and miR-145 in primary PAMs were increased along with time proceeding following PRRSV infection (Fig. 4A). The levels of four miRNAs could be upregulated approximately by 2-7 fold at $24 \mathrm{hpi}$, indicating that PRRSV infection is capable of inducing the generation of cellular let-7b, miR-26a, miR-34a and miR-145 during the early infection. The levels of let-7b, miR-26a, miR-34a and miR-145 in JXwn06-infected PAMs were significantly higher than those in HB-1/3.9-infected PAMs at 24 hpi $(\mathrm{P}<0.05$ or $\mathrm{P}<0.01)$, suggesting that the HP-PRRSV JXwn06 exhibits a higher ability of inducing let-7b, miR-26a, miR-34a 
A

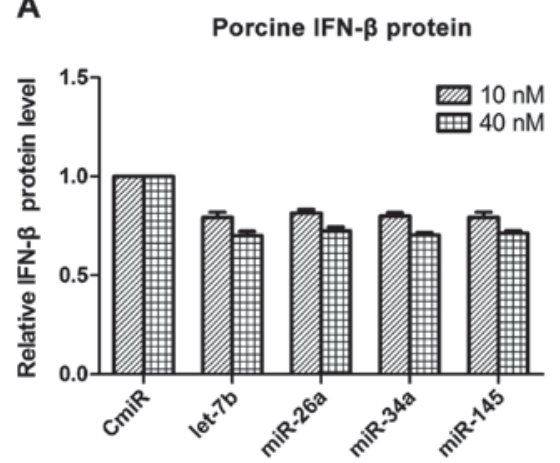

B

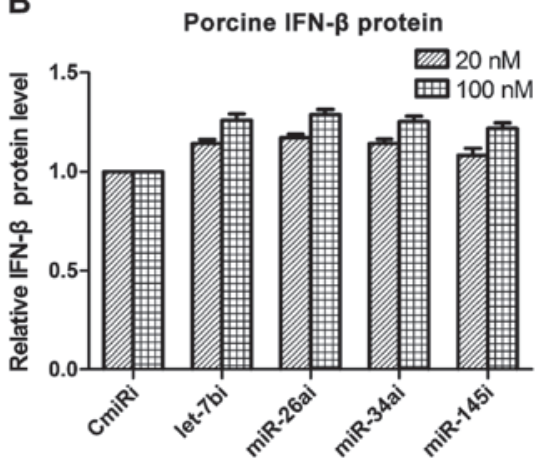

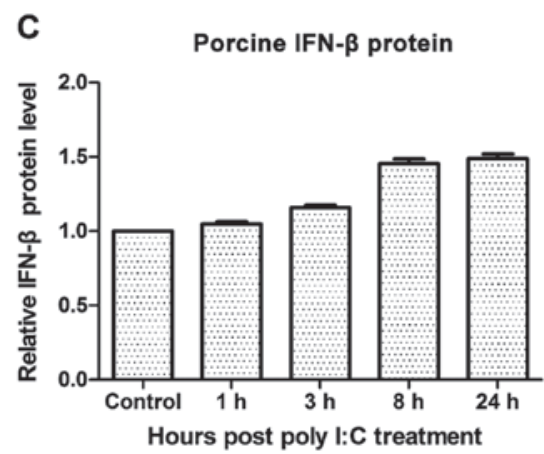

D
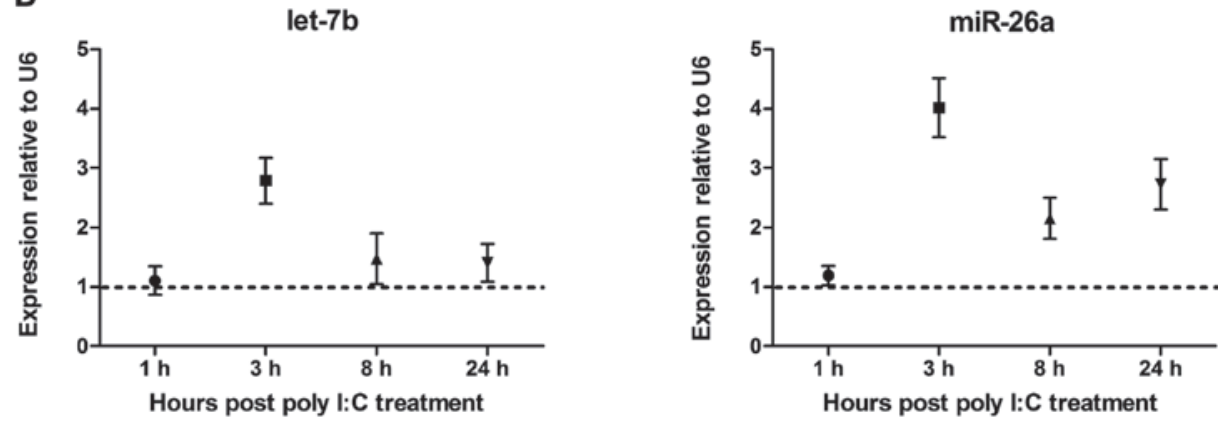

miR-34a
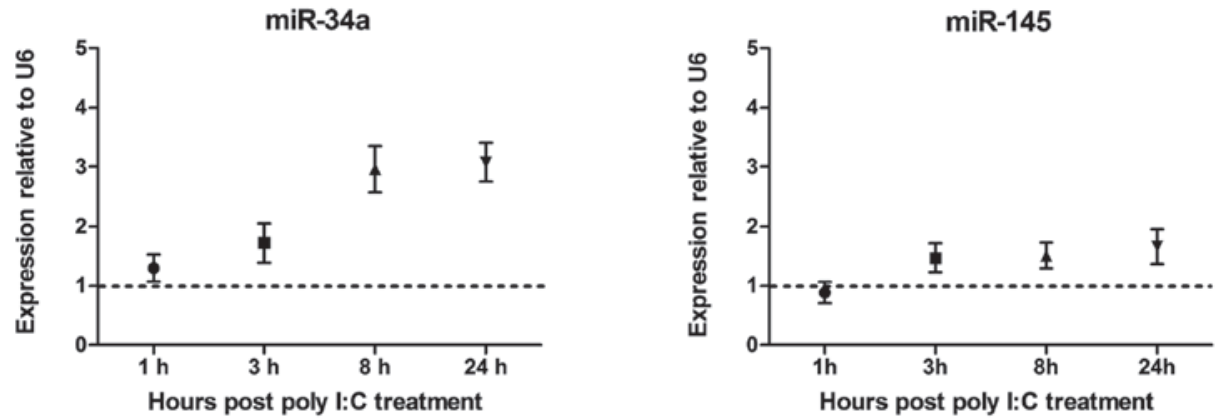

Figure 3. The mutual regulation between miRNAs and IFN- $\beta$ protein in PAMs. (A) 10 or 40 nM four miRNA mimics and negative control miRNA mimic (CmiR), and (B) 20 or $100 \mathrm{nM}$ four miRNA inhibitors and negative control miRNA inhibitor (CmiRi), were transfected transiently into primary PAMs, which were then stimulated by $50 \mu \mathrm{g} / \mathrm{ml}$ poly I:C for $24 \mathrm{~h}$. The protein expression levels of porcine IFN- $\beta$ in the supernatant of PAMs at 24 hpi were measured by ELISA. The IFN- $\beta$ protein expression could be suppressed by miRNA mimics (A) and the native endogenous miRNAs inhibition effect on IFN- $\beta$ protein expression could be relieved by miRNA inhibitors (B) in primary PAMs. (C) Primary PAMs were treated by $50 \mu \mathrm{g} / \mathrm{ml}$ poly I:C to stimulate the production of porcine IFN- $\beta$, whose protein levels were monitored by a commercial porcine IFN- $\beta$ ELISA kit at 1,38 and $24 \mathrm{~h}$ post-treatment. Mock-treated PAMs were used as control. (D) qRT-PCR analysis of miRNAs expression including let-7b, miR-26a, miR-34a and miR-145, in primary PAMs stimulated by 50 $\mu \mathrm{g} / \mathrm{ml}$ poly I:C at 1,3,8 and $24 \mathrm{~h}$ after treatment. The native IFN- $\beta$ protein induction stimulated by poly I:C could upregulate the expression of endogenous miRNAs in primary PAMs. The results were normalized to U6 expression and mock-treated controls according to $\Delta \Delta \mathrm{Ct}$ method. The data represent means \pm standard error of the mean of three independent experiments.

and miR-145 levels than low pathogenic PRRSV HB-1/3.9 during early infection.
The roles of miRNAs in PRRSV-mediated inhibition of porcine IFN- $\beta$ protein expression were further analyzed. 

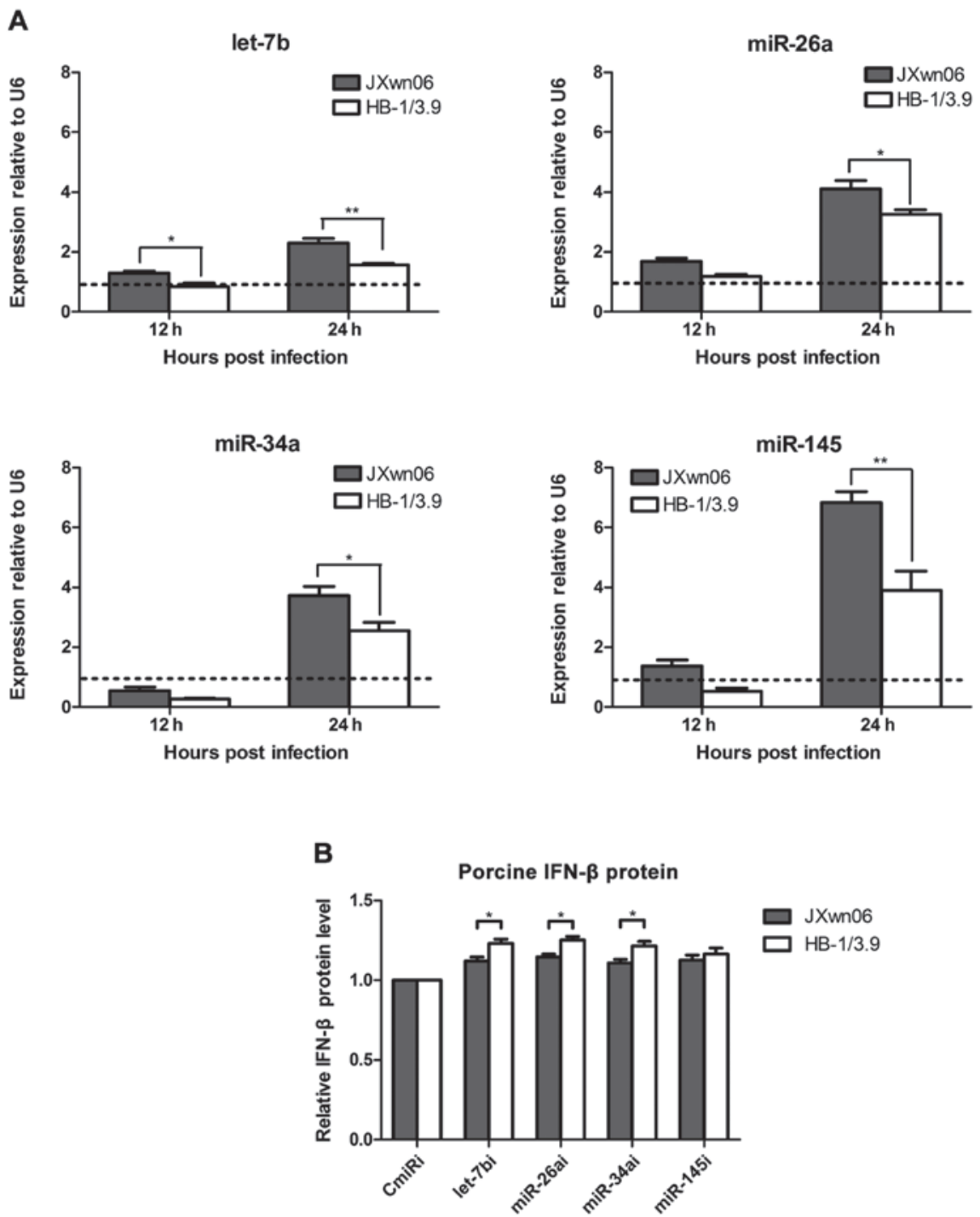

Figure 4. Inhibition of the production of IFN- $\beta$ protein in PRRSV-infected PAMs by upregulating cellular endogenous miRNAs. (A) qRT-PCR analysis of cellular endogenous miRNAs including let-7b, miR-26a, miR-34a and miR-145 in primary PAMs infected with JXwn06 or HB-1/3.9 at MOI of 1 at 12 and 24 hpi. Data were normalized to U6 expression and mock-infected controls according to $\Delta \Delta \mathrm{Ct}$ method. (B) Primary PAMs were transfected transiently with miRNA inhibitors including the antagonists of let-7b,26a,34a and miR-145 at $40 \mathrm{nM}$, and were then infected with JXwn06 or HB-1/3.9 at MOI of 1 at $24 \mathrm{~h}$ post-transfection. The protein expression of porcine IFN- $\beta$ in culture supernatants were measured by ELISA at 24 hpi. PAMs transfected with negative control miRNA inhibitor $(\mathrm{CmiRi})$ were used as control. The data represent means \pm standard error of the mean of three independent experiments $\left({ }^{*} \mathrm{P}<0.05{ }^{* *} \mathrm{P}<0.01\right)$.

Primary PAMs were transfected transiently with miRNA inhibitors including the antagonists of let-7b, miR-26a, miR-34a and miR-145 at a final concentration of $40 \mathrm{nM}$, and then infected with JXwn06 or HB-1/3.9 at MOI of 1 . The porcine IFN- $\beta$ protein expression was measured by ELISA kit. As shown in Fig. 4B, the protein expression of IFN- $\beta$ was upregulated in the miRNA inhibitor-transfected primary PAMs during PRRSV infection at $24 \mathrm{hpi}$, suggesting that the miRNA inhibitors including the antagonists of let-7b, miR-26a, miR-34a and miR-145, were able to relieve PRRSV-mediating inhibition of porcine IFN- $\beta$ protein expression to a certain extent. Compared with PRRSV-infected PAMs transfected with all miRNA inhibitors, but miRNA-145 inhibitor, the protein expression of IFN- $\beta$ in HB-1/3.9-infected PAMs were higher than that in JXwn06-infected PAMs $(\mathrm{P}<0.05)$, indicating that an equal amount of three miRNA inhibitors more effectively relieve low pathogenic PRRSV-mediated inhibition of porcine IFN- $\beta$ protein expression. This provided further evidence that
HP-PRRSV-mediating inhibition of porcine IFN- $\beta$ protein expression was stronger than low pathogenic PRRSV.

\section{Discussion}

Type I IFN-( $\alpha / \beta)$, most efficiently induced by leukocytes such as macrophages and dendritic cells, play multiple important functions in the innate and adaptive immune responses of host against various virus infections $(48,49)$. PAMs are important host target cells for PRRSV infection and replication. It is helpful for understanding the pathogenesis of PRRSV to investigate the interaction between the virus and its host cells. Previous studies have demonstrated that PRRSV is a poor inducer of type I IFN in vitro and in vivo $(1,50)$. In the present study, cellular miRNA-mediated regulation of IFN- $\beta$ protein expression was investigated in primary PAMs infected with PRRSV in vitro. Our results showed that the transcription level of IFN- $\beta$ mRNA was increased, but its protein 
expression was decreased in PRRSV-infected PAMs in the early stage, suggesting that there was a post-transcriptional inhibition mechanism of IFN- $\beta$ protein expression. Cellular miRNAs, including let-7b, miR-26a, miR-34a and miR-145, were capable of downregulating the porcine IFN- $\beta$ protein expression via targeting the binding sequences of IFN- $\beta$ 3'UTR, while porcine native IFN- $\beta$ protein could modulate cellular endogenous miRNAs expression using a negative feedback mechanism. Moreover, the four miRNAs were upregulated in PRRSV-infected PAMs, and their inhibitors could relieve PRRSV-mediated suppression of porcine IFN- $\beta$ protein expression.

Previous study has shown that post-transcriptional mechanism was involved in type I IFN induction in host cells during PRRSV infection (13). Here, we found that IFN- $\beta$ mRNA transcription could be activated, but its protein production was inhibited in PRRSV-infected PAMs during early infection, suggesting that PRRSV-mediated inhibition of IFN- $\beta$ protein production occurred in a post-transcriptional manner. Our results are consistent with previous studies using PAMs and porcine monocyte-derived dendritic cells $(14,20)$. Studies have demonstrated that some nsps and $\mathrm{N}$ protein of PRRSV were involved in inhibiting the IFN- $\beta$ promoter in MARC- 145 cells and human cells (21), it is debatable whether these results are similar in natural host cells of PRRSV, as the findings reported on mouse hepatitis virus (51).

Different pathogenic PRRSV isolates might be of distinct features in post-transcriptional control of IFN- $\beta$. Our results presented that HP-PRRSV JXwn06 and low pathogenic PRRSV HB-1/3.9 induced differential levels of IFN- $\beta$ protein expression in PAMs in vitro during early infection, and the post-transcriptional inhibition of IFN- $\beta$ induced by JXwn06 was stronger than that by HB-1/3.9 at the same initial viral infectious dose, indicating that different pathogenic PRRSV strains have different ability in the regulation of IFN- $\beta$ protein expression in vitro. Our data are consistent with the study that the IFN phenotypes induced by PRRSV field isolates were distinct in vitro (20), and is similar to the study that the Chinese HP-PRRSV isolate displayed more inhibitory effect than PRRSV strain VR-2332 in the suppression of CpG-ODN-induced IFN- $\alpha$ responses by enriched plasmacytoid dendritic cells (pDC) (50). The differential IFN- $\beta$ response of PAMs to different pathogenic PRRSV strains may be due to the viral distinct characteristics or variation in efficiency of viral infection and replication. Chinese HP-PRRSV JXwn06 possess higher replication efficiency and infection capacity than low pathogenic PRRSV HB-1/3.9 in PAMs in vitro at the same initial infectious dose [data not shown, as our similar recent study described by Li et al (52)], which may contribute to the stronger post-transcriptional inhibition effect of porcine IFN- $\beta$ protein expression by HP-PRRSV. Since IFN- $\beta$ is the first batch of host genes expression after PRRSV infection and a few fold changes in its protein level leads to almost amplifying the IFN- $\beta$ effect thousands of times by the signaling cascades (47), the differences between HP-PRRSV and low pathogenic PRRSV in the inhibition of IFN- $\beta$ induction in PAMs during early infection might correlate with their different virulence and replication efficiency in vivo. Compared with wild-type viruses, UV- and heat-inactivated PRRSV fail to suppress type I IFN production in vitro induced by TGEV or poly $\mathrm{I}: \mathrm{C}$, indicating that virus replication and cytopathogenicity are essential for PRRSV to inhibit the expression of type I IFN $(1,53)$. Previous studies showed that type I IFN induction correlated with the virulence of some viruses (54). Additionally, our recent study showed that HP-PRRSV and low pathogenic PRRSV elicited differential TNF- $\alpha$ subtype production in vitro, suggesting that different pathogenic isolates of PRRSV might possess different capacity to induce other cytokines in vitro (43).

Post-transcriptional control of the IFN system is involved in multiple levels of regulation including mRNA stability, alternative splicing, translation, and post-translational effects (55). miRNAs have been shown to play important roles in regulating the IFN- $\alpha / \beta$ response at the post-transcriptional level $(23,56)$. Given that there is a subset of IFN- $\alpha$ and a single IFN- $\beta$ subtype, and neither a single IFN- $\alpha$ subtype nor all IFN- $\alpha$ can be activated by reported stimuli (57), we chose porcine IFN- $\beta$ to investigate the interaction between miRNAs and type I IFN. In the present study, our results indicated that porcine IFN- $\beta$ protein expression in primary PAMs could be downregulated by cellular let-7b, miR-26a, miR-34a and miR-145 which are capable of targeting the binding sequences of porcine IFN- $\beta$ 3'UTR. Similar phenomenon has been described by an early study using primary primate macrophages (47). It is possible that porcine IFN- $\beta$ response is modulated at different levels in primary PAMs, even more miRNAs may be involved in this regulation. Therefore, further studies are required to discover additional mechanisms or miRNAs in primary PAMs.

PRRSV has evolved several strategies to evade and antagonize anti-viral immune responses of host including using miRNAs (58). Data presented in this study showed that cellular endogenous miRNAs including let-7b, miR-26a, miR-34a and miR-145, were upregulated in PAMs during early infection of PRRSV, and miRNA antagonists enhanced the production of porcine IFN- $\beta$ protein, implying that PRRSV may utilize the cellular miRNAs including let-7b, miR-26a, miR-34a and miR-145, to negatively regulate porcine IFN- $\beta$ response post-transcriptionally, and further to facilitate virus replication, since porcine IFN- $\beta$ has been shown to protect PAMs from PRRSV infection (59). Interestingly, similar to Li et al (35), a recent study has demonstrated that miR-26a overexpressed in MARC-145 cells triggered IFN- $\beta$ signaling pathway during PRRSV infection, but the protein expression of IFN- $\beta$ was not increased, indicating that miR-26a may modulate the IFN- $\beta$ expression post-transcriptionally (34). Similarly, since miR-373 could downregulate the transcription of IFN- $\beta$ via targeting cellular factors such as NFIA, NFIB, IRAK1, IRAK4 and IRF1, PRRSV could upregulate the expression of miR-373 by elevating Sp1 expression in MARC145 cells during viral infection (33), which suggests that PRRSV is capable of altering cellular miRNA expression to modulate immune response and to facilitate the viral immune escape. Consistent with this possibility, PRRSV infection can not only suppress host miR-125b and poly I:C-induced miR-23, but also elevate miR-146a, suggesting that these miRNA-mediated regulations might be strategies for PRRSV to evade the type I IFN response $(30,33,60,61)$. Supporting this, many viruses could induce miRNAs to escape from immune responses such as enterovirus 71-induced miR-146a in the mouse model, HCV-induced miR-21 in hepatocytes and 
respiratory syncytial virus-induced let-7b in DCs (62-64). Our present data suggested that porcine IFN- $\beta$ production could be augmented by suppressing the expression of endogenous miRNAs including let-7b, miR-26a, miR-34a and miR-145, which could be a new approach in future exploration to prevent PRRSV infection.

In conclusion, our findings for the first time demonstrated that the protein expression of IFN- $\beta$ could be suppressed by porcine cellular miRNAs, including let-7b, miR-26a, miR-34a and miR-145, in PAMs by directly targeting the sequences within the porcine IFN- $\beta$ 3'UTR. PRRSV could inhibit the IFN- $\beta$ protein expression in PAMs by means of upregulating the levels of the four porcine miRNAs during early viral infection, which may contribute to the post-transcriptional control of IFN- $\beta$ in PRRSV-infected host cells and to the establishment of viral persistent infection. The molecular mechanism by which PRRSV upregulates cellular miRNAs in host cells will be explored in the future.

\section{Acknowledgements}

The present study was supported by The Major Program of National Natural Science Funds from The National Natural Science Foundation of China (31490603), The National Key Basic Research PlanGrant (2014CB542700) from the Chinese Ministry of Science and Technology, and the Earmarked Fund for Modern Agro-industry Technology Research System of China (CARS-35) from the Chinese Ministry of Agriculture of People's Republic of China.

\section{References}

1. Albina E: Epidemiology of porcine reproductive and respiratory syndrome (PRRS): An overview. Vet Microbiol 55: 309-316, 1997.

2. Rossow KD: Porcine reproductive and respiratory syndrome. Vet Pathol 35: 1-20, 1998.

3. Keffaber K: Reproductive failure of unknown etiology. Am Assoc Swine Prac News 1: 1-9, 1989.

4. Cho JG and Dee SA: Porcine reproductive and respiratory syndrome virus. Theriogenology 66: 655-662, 2006.

5. Tian K, Yu X, Zhao T, Feng Y, Cao Z, Wang C, Hu Y, Chen X, $\mathrm{Hu}$ D, Tian X, et al: Emergence of fatal PRRSV variants: Unparalleled outbreaks of atypical PRRS in China and molecular dissection of the unique hallmark. PLoS One 2: e526, 2007.

6. Zhou L and Yang H: Porcine reproductive and respiratory syndrome in China. Virus Res 154: 31-37, 2010.

7. Cavanagh D: Nidovirales: A new order comprising Coronaviridae and Arteriviridae. Arch Virol 142: 629-633, 1997.

8. Conzelmann KK, Visser N, Van Woensel P and Thiel HJ: Molecular characterization of porcine reproductive and respiratory syndrome virus, a member of the arterivirus group. Virology 193: 329-339, 1993.

9. Meulenberg JJ, Hulst MM, de Meijer EJ, Moonen PL, den Besten A, de Kluyver EP, Wensvoort G and Moormann RJ: Lelystad virus, the causative agent of porcine epidemic abortion and respiratory syndrome (PEARS), is related to LDV and EAV. Virology 192: 62-72, 1993.

10. Mardassi H, Mounir S and Dea S: Identification of major differences in the nucleocapsid protein genes of a Québec strain and European strains of porcine reproductive and respiratory syndrome virus. J Gen Virol 75: 681-685, 1994.

11. Meng XJ, Paul PS, Halbur PG and Lum MA: Phylogenetic analyses of the putative M (ORF 6) and $\mathrm{N}$ (ORF 7) genes of porcine reproductive and respiratory syndrome virus (PRRSV): Implication for the existence of two genotypes of PRRSV in the USA and Europe. Arch Virol 140: 745-755, 1995.

12. Nelson EA, Christopher-Hennings J, Drew T, Wensvoort G, Collins JE and Benfield DA: Differentiation of U.S. and European isolates of porcine reproductive and respiratory syndrome virus by monoclonal antibodies. J Clin Microbiol 31: 3184-3189, 1993.
13. Wang $X$ and Christopher-Hennings J: Post-transcriptional control of type I interferon induction by porcine reproductive and respiratory syndrome virus in its natural host cells. Viruses 4: 725-733, 2012.

14. Zhang H, Guo X, Nelson E, Christopher-Hennings J and Wang X: Porcine reproductive and respiratory syndrome virus activates the transcription of interferon alpha/beta (IFN- $\alpha / \beta)$ in monocyte-derived dendritic cells (Mo-DC). Vet Microbiol 159: 494-498, 2012.

15. Albina E, Carrat $\mathrm{C}$ and Charley B: Interferon-alpha response to swine arterivirus (PoAV), the porcine reproductive and respiratory syndrome virus. J Interferon Cytokine Res 18: 485-490, 1998 .

16. Buddaert W, Van Reeth $\mathrm{K}$ and Pensaert M: In vivo and in vitro interferon (IFN) studies with the porcine reproductive and respiratory syndrome virus (PRRSV). Adv Exp Med Biol 440: 461-467, 1998.

17. Calzada-Nova G, Schnitzlein WM, Husmann RJ and Zuckermann FA: North American porcine reproductive and respiratory syndrome viruses inhibit type I interferon production by plasmacytoid dendritic cells. J Virol 85: 2703-2713, 2011.

18. Miller LC, Laegreid WW, Bono JL, Chitko-McKown CG and Fox JM: Interferon type I response in porcine reproductive and respiratory syndrome virus-infected MARC-145 cells. Arch Virol 149: 2453-2463, 2004.

19. Chung HK, Lee JH, Kim SH and Chae C: Expression of interferon-alpha and $\mathrm{Mx} 1$ protein in pigs acutely infected with porcine reproductive and respiratory syndrome virus (PRRSV). J Comp Pathol 130: 299-305, 2004.

20. Lee SM, Schommer SK and Kleiboeker SB: Porcine reproductive and respiratory syndrome virus field isolates differ in in vitro interferon phenotypes. Vet Immunol Immunopathol 102: 217-231, 2004.

21. Sun Y, Han M, Kim C, Calvert JG and Yoo D: Interplay between interferon-mediated innate immunity and porcine reproductive and respiratory syndrome virus. Viruses 4: 424-446, 2012.

22. Lin S and Gregory RI: MicroRNA biogenesis pathways in cancer. Nat Rev Cancer 15: 321-333, 2015.

23. Forster SC, Tate MD and Hertzog PJ: MicroRNA as type I interferon-regulated transcripts and modulators of the innate immune response. Front Immunol 6: 334, 2015.

24. Cullen BR: MicroRNAs as mediators of viral evasion of the immune system. Nat Immunol 14: 205-210, 2013

25. Skalsky RL and Cullen BR: Viruses, microRNAs, and host interactions. Annu Rev Microbiol 64: 123-141, 2010.

26. Cong P, Xiao S, Chen Y, Wang L, Gao J, Li M, He Z, Guo Y, Zhao G, Zhang X, et al: Integrated miRNA and mRNA transcriptomes of porcine alveolar macrophages (PAM cells) identifies strain-specific miRNA molecular signatures associated with H-PRRSV and N-PRRSV infection. Mol Biol Rep 41: 5863-5875, 2014.

27. Li J, Chen Z, Zhao J, Fang L, Fang R, Xiao J, Chen X, Zhou A, Zhang Y, Ren L, et al: Difference in microRNA expression and editing profile of lung tissues from different pig breeds related to immune responses to HP-PRRSV. Sci Rep 5: 9549, 2015.

28. Wang C, Zhang Y, Luo J, Ding H, Liu S, Amer S, Xie L, Lyv W, Su W, Li M, et al: Identification of miRNomes reveals ssc-miR-30d-R_1 as a potential therapeutic target for PRRS viral infection. Sci Rep 6: 24854, 2016.

29. Li L, Gao F, Jiang Y, Yu L, Zhou Y, Zheng H, Tong W, Yang S, Xia T, Qu Z, et al: Cellular miR-130b inhibits replication of porcine reproductive and respiratory syndrome virus in vitro and in vivo. Sci Rep 5: 17010, 2015.

30. Wang D, Cao L, Xu Z, Fang L, Zhong Y, Chen Q, Luo R, Chen H, Li K and Xiao S: MiR-125b reduces porcine reproductive and respiratory syndrome virus replication by negatively regulating the NF- $\kappa$ B pathway. PLoS One 8: e55838, 2013.

31. Xiao S, Wang X, Ni H, Li N, Zhang A, Liu H, Pu F, Xu L, Gao J, Zhao Q, et al: MicroRNA miR-24-3p promotes porcine reproductive and respiratory syndrome virus replication through suppression of heme oxygenase-1 expression. J Virol 89: 4494-4503, 2015

32. Zhang Q, Huang C, Yang Q, Gao L, Liu HC, Tang J and Feng WH: MicroRNA-30c modulates type I IFN responses to facilitate porcine reproductive and respiratory syndrome virus infection by targeting JAK1. J Immunol 196: 2272-2282, 2016.

33. Chen J, Shi X, Zhang X, Wang A, Wang L, Yang Y, Deng R and Zhang GP: MicroRNA-373 facilitated the replication of porcine reproductive and respiratory syndrome virus by its negative regulation of type I interferon Induction. J Virol 91: e01311-e01316, 2017. 
34. Jia X, Bi Y, Li J, Xie Q, Yang H and Liu W: Cellular microRNA miR-26a suppresses replication of porcine reproductive and respiratory syndrome virus by activating innate antiviral immunity. Sci Rep 5: 10651, 2015.

35. Li L, Wei Z, Zhou Y, Gao F, Jiang Y, Yu L, Zheng H, Tong W, Yang S, Zheng H, et al: Host miR-26a suppresses replication of porcine reproductive and respiratory syndrome virus by upregulating type I interferons. Virus Res 195: 86-94, 2015.

36. He D, Overend C, Ambrogio J, Maganti RJ, Grubman MJ and Garmendia AE: Marked differences between MARC-145 cells and swine alveolar macrophages in IFN $\beta$-induced activation of antiviral state against PRRSV. Vet Immunol Immunopathol 139: 57-60, 2011.

37. Ait-Ali T, Wilson AD, Westcott DG, Clapperton M, Waterfall M, Mellencamp MA, Drew TW, Bishop SC and Archibald AL: Innate immune responses to replication of porcine reproductive and respiratory syndrome virus in isolated Swine alveolar macrophages. Viral Immunol 20: 105-118, 2007.

38. Zhang H, Guo X, Ge X, Chen Y, Sun Q and Yang H: Changes in the cellular proteins of pulmonary alveolar macrophage infected with porcine reproductive and respiratory syndrome virus by proteomics analysis. J Proteome Res 8: 3091-3097, 2009.

39. Zhou L, Zhang J, Zeng J, Yin S, Li Y, Zheng L, Guo X, Ge X and Yang H: The 30-amino-acid deletion in the Nsp2 of highly pathogenic porcine reproductive and respiratory syndrome virus emerging in China is not related to its virulence. J Virol 83 : 5156-5167, 2009

40. John B, Enright AJ, Aravin A, Tuschl T, Sander C and Marks DS: Human MicroRNA targets. PLoS Biol 2: e363, 2004.

41. Rehmsmeier M, Steffen P, Hochsmann M and Giegerich R: Fast and effective prediction of microRNA/target duplexes. RNA 10 $1507-1517,2004$

42. Kertesz M, Iovino N, Unnerstall U, Gaul U and Segal E: The role of site accessibility in microRNA target recognition. Nat Genet 39: 1278-1284, 2007.

43. He Q, Li Y, Zhou L, Ge X, Guo X and Yang H: Both Nsp1 $\beta$ and Nspl1 are responsible for differential TNF- $\alpha$ production induced by porcine reproductive and respiratory syndrome virus strains with different pathogenicity in vitro. Virus Res 201: 32-40, 2015.

44. Guo XK, Zhang Q, Gao L, Li N, Chen XX and Feng WH: Increasing expression of microRNA 181 inhibits porcine reproductive and respiratory syndrome virus replication and has implications for controlling virus infection. J Virol 87 : $1159-1171,2013$

45. Loving CL, Brockmeier SL and Sacco RE: Differential type I interferon activation and susceptibility of dendritic cell populations to porcine arterivirus. Immunology 120: 217-229, 2007.

46. Nygard AB, Jørgensen CB, Cirera S and Fredholm M: Selection of reference genes for gene expression studies in pig tissues using SYBR green qPCR. BMC Mol Biol 8: 67, 2007.

47. Witwer KW, Sisk JM, Gama L and Clements JE: MicroRNA regulation of IFN-beta protein expression: Rapid and sensitive modulation of the innate immune response. J Immunol 184 2369-2376, 2010.

48. Biron CA: Role of early cytokines, including alpha and beta interferons (IFN-alpha/beta), in innate and adaptive immune responses to viral infections. Semin Immunol 10: 383-390, 1998

49. Theofilopoulos AN, Baccala R, Beutler B and Kono DH: Type I interferons (alpha/beta) in immunity and autoimmunity. Annu Rev Immunol 23: 307-336, 2005.
50. Baumann A, Mateu E, Murtaugh MP and Summerfield A: Impact of genotype 1 and 2 of porcine reproductive and respiratory syndrome viruses on interferon- $\alpha$ responses by plasmacytoid dendritic cells. Vet Res (Faisalabad) 44: 33, 2013.

51. Rose KM and Weiss SR: Murine coronavirus cell type dependent interaction with the type I interferon response. Viruses 1: 689-712, 2009.

52. Li Y, Zhou L, Zhang J, Ge X, Zhou R, Zheng H, Geng G, Guo X and Yang H: Nsp9 and Nsp10 contribute to the fatal virulence of highly pathogenic porcine reproductive and respiratory syndrome virus emerging in China. PLoS Pathog 10: e1004216, 2014.

53. Carrigan DR and Knox KK: Identification of interferon-resistant subpopulations in several strains of measles virus: Positive selection by growth of the virus in brain tissue. J Virol 64: 1606-1615, 1990

54. Marcus PI, Rojek JM and Sekellick MJ: Interferon induction and/or production and its suppression by influenza A viruses. J Virol 79: 2880-2890, 2005.

55. Khabar KS and Young HA: Post-transcriptional control of the interferon system. Biochimie 89: 761-769, 2007.

56. Sedger LM: microRNA control of interferons and interferon induced anti-viral activity. Mol Immunol 56: 781-793, 2013.

57. Hertzog PJ and Williams BR: Fine tuning type I interferon responses. Cytokine Growth Factor Rev 24: 217-225, 2013.

58. Huang C, Zhang Q and Feng WH: Regulation and evasion of antiviral immune responses by porcine reproductive and respiratory syndrome virus. Virus Res 202: 101-111, 2015.

59. Overend C, Mitchell R, He D, Rompato G, Grubman MJ and Garmendia AE: Recombinant swine beta interferon protects swine alveolar macrophages and MARC-145 cells from infection with Porcine reproductive and respiratory syndrome virus. J Gen Virol 88: 925-931, 2007.

60. A Hicks J, Yoo D and Liu HC: Characterization of the microRNAome in porcine reproductive and respiratory syndrome virus infected macrophages. PLoS One 8: e82054, 2013.

61. Zhang Q, Guo XK, Gao L, Huang C, Li N, Jia X, Liu W and Feng WH: MicroRNA-23 inhibits PRRSV replication by directly targeting PRRSV RNA and possibly by upregulating type I interferons. Virology 450-451: 182-195, 2014.

62. Chen Y, Chen J, Wang H, Shi J, Wu K, Liu S, Liu Y and Wu J. HCV-induced miR-21 contributes to evasion of host immune system by targeting MyD88 and IRAK1. PLoS Pathog 9: e1003248, 2013.

63. Ho BC, Yu IS, Lu LF, Rudensky A, Chen HY, Tsai CW, Chang YL, Wu CT, Chang LY, Shih SR, et al: Inhibition of miR-146a prevents enterovirus-induced death by restoring the production of type I interferon. Nat Commun 5: 3344, 2014.

64. Thornburg NJ, Hayward SL and Crowe JE Jr: Respiratory syncytial virus regulates human microRNAs by using mechanisms involving beta interferon and NF-кB. MBio 3: e002, 2012.

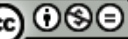

This work is licensed under a Creative Commons Attribution-NonCommercial-NoDerivatives 4.0 International (CC BY-NC-ND 4.0) License. 